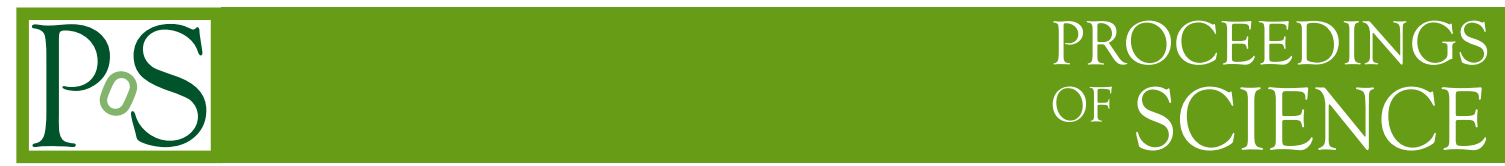

\title{
Studies of charm mixing and CP violation at $\mathrm{LHCb}$
}

\author{
Jordi Garra Tico* \\ University of Cambridge \\ E-mail: jordix@hep.phy.cam.ac.uk
}

The LHCb detector at CERN is an LHC experiment focused on the study of beauty and charm physics. This document presents an LHCb result devoted to the measurement of the mixing parameter $y_{C P}$, related to the mixing parameter $y$, and three searches for charm $C P$ violation. Sensitivity to both mixing and $C P$ violation in LHCb has become very promising.

36th International Conference on High Energy Physics

4-11 July 2012

Melbourne, Australia

${ }^{*}$ Speaker. 


\section{Introduction to mixing of neutral $D$ mesons}

For a system of a pair of neutral $D$ mesons, different neutral states are relevant to the discussion of different processes:

- The two flavor eigenstates, $\left|D^{0}\right\rangle$ and $\left|\bar{D}^{0}\right\rangle$, have a definite quark flavor content and are those relevant to particle production and decay processes. They are mixed with each other as they propagate through space and time.

- The two Hamiltonian eigenstates, $\left|D_{1}\right\rangle$ and $\left|D_{2}\right\rangle$, have definite mass and lifetime, and they propagate through space and time in a definite way. The Hamiltonian eigenstates are written in the flavor basis as

$$
\left|D_{1,2}\right\rangle=p\left|D^{0}\right\rangle \pm q\left|\bar{D}^{0}\right\rangle
$$

- If $C P$ were preserved, the Hamiltonian eigenstates would also be $C P$ eigenstates, namely $\left|D_{+}\right\rangle$and $\left|D_{-}\right\rangle$.

For the system of the two neutral kaons, it is relevant to observe that their mass eigenstates have different average lifetimes, although similar masses, so it is convenient to define their states as $K_{L}^{0}$ and $K_{S}^{0}$ for the long-lived and short-lived states, respectively. However, for neutral $D$ mesons, the mixing rate is slower than the decay rate, so the flavor eigenstates are the most convenient basis for them.

An arbitrary linear combination of the neutral $D$ flavor eigenstates, $a\left|D^{0}\right\rangle+b\left|\bar{D}^{0}\right\rangle$, evolves in time according to the time-dependent Schrödinger equation

$$
i \frac{d}{d t}\left(\begin{array}{l}
a \\
b
\end{array}\right)=\mathscr{H}\left(\begin{array}{l}
a \\
b
\end{array}\right) \equiv\left(M-\frac{i}{2} \Gamma\right)\left(\begin{array}{l}
a \\
b
\end{array}\right),
$$

where $M$ and $\Gamma$ are $2 \times 2$ Hermitian matrices, but the effective Hamiltonian $\mathscr{H}$ is not. $\left|D_{1}\right\rangle$ and $\left|D_{2}\right\rangle$ are the eigenstates of $\mathscr{H}$, with eigenvalues $\left(m_{1}+\frac{i}{2} \Gamma_{1}\right)$ and $\left(m_{2}+\frac{i}{2} \Gamma_{2}\right)$, respectively.

The mixing parameters $x$ and $y$ are defined as

$$
\Gamma=\frac{\Gamma_{1}+\Gamma_{2}}{2}, \quad x=\frac{m_{1}-m_{2}}{\Gamma}, \quad y=\frac{\Gamma_{1}-\Gamma_{2}}{2 \Gamma},
$$

and the time evolution of the elements of any $D$ meson basis can be consistently expressed with respect to them.

\section{Introduction to $C P$ violation in $D$ meson decays}

The possible manifestations of $C P$ violation can be classified in a model-independent way:

- $C P$ violation in the decay occurs in decays of both charged and neutral mesons. It occurs when the amplitude of a decay is different from that of the decay of its $C P$ conjugate.

- $C P$ violation in the mixing occurs in neutral meson decays, when the Hamiltonian eigenstates cannot be chosen to be $C P$ eigenstates as well.

- $C P$ violation in the interference between decays with and without mixing occurs in decays into final states that are common to $\left|D^{0}\right\rangle$ and $\left|\bar{D}^{0}\right\rangle$. 


\section{Standard model predictions of $D$ meson mixing and CPV}

Mixing in the standard model originates from short and long distance contributions. Short distance contributions come from box diagrams with quarks and $W^{ \pm}$bosons. The standard model predicts small mixing effects because $b$ quarks are CKM suppressed and $s$ and $d$ quarks are GIM suppressed [1]. The main short distance contribution is to the $x$ mixing parameter. Long distance contributions come from loop diagrams with hadronic intermediate states. These contributions are expected to be dominant, but still small. Since they are not perturbative, they are difficult to estimate, but predictions exist [2,3] that bound $x$ and $y$ in the range [0.001,0.01], with $|x|<|y|$.

New physics could arise through new particles in loops. For example, if $|x|$ was found to be much larger than $|y|$, this could be a hint of new physics.

The standard model predicts CPV in the $D$ sector to be $\sim 10^{-3}$. If CPV was to be found with the current sensitivity $\left(\sim 10^{-2}\right)$, this would also be a hint of new physics [3].

\section{Mixing and $C P$ violation searches}

Mixing has been studied using a variety of suppressed hadronic decays: in the time dependence of the wrong sign events in $D^{0} \rightarrow K^{+} \pi^{-}[4,5,6]$, in the ratio of lifetimes of $D^{0} \rightarrow K^{+} K^{-}$and $D^{0} \rightarrow \pi^{+} \pi^{-}$events with respect to $D^{0} \rightarrow K^{-} \pi^{+}$events [7, 8, 9], and in a $D^{0}$ time-dependent amplitude analysis of $D^{0} \rightarrow K^{+} \pi^{-} \pi^{0}$ events [10]. Mixing has also been studied in semileptonic events $[11,12,13]$ by the BaBar and Belle collaborations, and in a time-dependent amplitude analysis of $K_{s} \pi^{+} \pi^{-}$events $[14,15]$.

$D$ mesons are tagged at production by means of $D^{\star+} \rightarrow D^{0} \pi_{s}^{+}$and its conjugate, where the charge of the low momentum pion $\pi_{s}^{ \pm}$tags the flavor of the produced $D$ meson.

$C P$ violation has been studied in single Cabibbo-suppressed decays with $C P$ even final states $D^{0} \rightarrow \pi^{+} \pi^{-}$and $D^{0} \rightarrow K^{+} K^{-}[16,17]$ and $D^{0} \rightarrow \pi^{+} \pi^{-} \pi^{0}[18,19]$ and $D^{0} \rightarrow K^{+} K^{-} \pi^{0}$ [18]. The standard model predicts $C P$ violation in these modes to be $\sim 10^{-4}-10^{-5}$, so evidence of $C P$ violation in them would be a sign of physics beyond the standard model [3]. $C P$ violation has also been studied in $D^{0} \rightarrow K^{+} \pi^{-} \pi^{0}$ events $[10,20]$ and in $D^{0} \rightarrow K^{+} K^{-} \pi^{+} \pi^{-}$decays.

\section{LHCb results}

The analyses presented in this document are devoted to measure the mixing parameters and $C P$ violation effects by means of different techniques. In all analyses that require $D$ meson flavor tagging at production, the $D^{\star \pm} \rightarrow \tilde{D}^{0} \pi^{ \pm}$decay is used, where $\tilde{D}^{0}$ represents either a $D^{0}$ or a $\bar{D}^{0}$, and the charge of the soft pion determines its flavor.

\subsection{Measurement of $y_{C P}$ and $A_{\Gamma}$}

The $y_{C P}$ mixing parameter is the equivalent of the $y$ mixing parameter under the assumption that $C P$ symmetry is conserved. It can be obtained from lifetime measurements in $D$ meson twobody decays,

$$
y_{C P}=\frac{\tau\left(D^{0} \rightarrow K^{-} \pi^{+}\right)}{\tau\left(D^{0} \rightarrow K^{-} K^{+}\right)}-1 .
$$


The $A_{\Gamma} C P$ violation parameter is the relative difference between the $D^{0}$ and $\bar{D}^{0}$ decay rates, which is evaluated as

$$
A_{\Gamma}=\frac{\tau\left(\bar{D}^{0} \rightarrow K^{-} K^{+}\right)-\tau\left(D^{0} \rightarrow K^{-} K^{+}\right)}{\tau\left(\bar{D}^{0} \rightarrow K^{-} K^{+}\right)+\tau\left(D^{0} \rightarrow K^{-} K^{+}\right)} .
$$

These two measurements require a good reconstruction of the $D$ meson lifetime and, in particular, the lifetime biases introduced by the trigger are accounted for with the so called swimming technique [21,22], which evaluates the lifetime acceptance from data by means of faking a different position of the primary vertex and running the trigger algorithms on it.

The LHCb result with $29 \mathrm{pb}^{-1}$ of data measured in 2010 at a center of mass energy of $7 \mathrm{TeV}$ is [23]

$$
\begin{aligned}
y_{C P} & =[5.5 \pm 6.3(\text { stat }) \pm 4.1(\text { syst })] \cdot 10^{-3}, \\
A_{\Gamma} & =[-5.9 \pm 5.9(\text { stat }) \pm 2.1(\text { syst })] \cdot 10^{-3} .
\end{aligned}
$$

\subsection{Measurement of $C P V$ in $D \rightarrow h^{+} h^{-}$decays}

The $C P$ asymmetry of a $D$ meson decay to a $C P$ eigenstate $f$ is given by

$$
A_{C P}(f, t)=\frac{\Gamma\left(D^{0}(t) \rightarrow f\right)-\Gamma\left(\bar{D}^{0}(t) \rightarrow f\right)}{\Gamma\left(D^{0}(t) \rightarrow f\right)+\Gamma\left(\bar{D}^{0}(t) \rightarrow f\right)},
$$

which in general depends on the $C P$ eigenstate $f$ and the $D$ meson lifetime $t$. The time integrated $\triangle A_{C P}$ parameter is defined as the time integrated difference of $C P$ asymemtries between $D$ meson decays to the $C P$ eigenstates $K K$ and $\pi \pi$,

$$
\Delta A_{C P}=A_{C P}(K K)-A_{C P}(\pi \pi) .
$$

$D^{\star \pm}$ production asymmetry and soft pion and $D$ meson detection asymmetries cancel at first order. Therefore, to a good approximation,

$$
\Delta A_{C P}=A_{C P}(K K)-A_{C P}(\pi \pi)=A_{\text {raw }}(K K)-A_{\text {raw }}(\pi \pi),
$$

with

$$
A_{\text {raw }}=\frac{N\left(D^{\star+} \rightarrow D^{0}(f) \pi_{s}^{+}\right)-N\left(D^{\star-} \rightarrow \bar{D}^{0}(f) \pi_{s}^{-}\right)}{N\left(D^{\star+} \rightarrow D^{0}(f) \pi_{s}^{+}\right)+N\left(D^{\star-} \rightarrow \bar{D}^{0}(f) \pi_{s}^{-}\right)} .
$$

Data are split in 216 bins of kinematics (transverse momentum $p_{t}$ and pseudorapidity $\eta$ ), LHCb magnet polarity and running periods. The yields $N$ are obtained from fits to $\delta m=m_{D^{\star}}-$ $m_{D}-m_{\pi}$ in these bins, and the measured asymmetries are consistent in all of them.

The time integrated asymmetry $\triangle A_{C P}$ contains contributions from both direct $\left(a_{C P}^{\mathrm{dir}}\right)$ and indirect $\left(a_{C P}^{\text {ind }}\right) C P$ violation, which can be expressed as a function of the average reconstructed $D$ lifetime difference $\Delta\langle t\rangle$ in the $K K$ and $\pi \pi$ samples [24, 25],

$$
\Delta A_{C P}=\left[a_{C P}^{\mathrm{dir}}(K K)-a_{C P}^{\mathrm{dir}}(\pi \pi)\right]+\frac{\Delta\langle t\rangle}{\tau_{D}} a_{C P}^{\mathrm{ind}}
$$

The LHCb measurement with $0.62 \mathrm{fb}^{-1}$ of data taken in 2011 shows the first evidence of $C P$ violation in the charm sector [26],

$$
\Delta A_{C P}=[-0.82 \pm 0.21(\text { stat }) \pm 0.11(\text { syst })] \cdot 10^{-2},
$$


also confirmed by CDF with $9.7 \mathrm{fb}^{-1}$ of collisions taken from 2002 to 2011 [27, 28],

$$
\Delta A_{C P}=[-0.62 \pm 0.21(\text { stat }) \pm 0.10(\text { syst })] \cdot 10^{-2} .
$$

\subsection{Search for $C P$ violation in $D^{+} \rightarrow K^{-} K^{+} \pi^{+}$decays}

This $C P$ violation measurement is a model independent search based on the distribution of the asymmetry significance in bins $k$ of the phase space, defined as

$$
S_{C P}^{k}=\frac{N_{k}\left(D^{+}\right)-\alpha N_{k}\left(D^{-}\right)}{\sqrt{N_{k}\left(D^{+}\right)+\alpha^{2} N_{k}\left(D^{-}\right)}},
$$

where the factor $\alpha$, defined as

$$
\alpha=\frac{N_{\text {total }}\left(D^{+}\right)}{N_{\text {total }}\left(D^{-}\right)}
$$

removes any global asymmetries.

If $C P$ is conserved, the distribution of all the $S_{C P}^{k}$ is expected to be normal (Gaussian centered at $\mu=0$ and with width $\sigma=1$ ). Monte Carlo studies show that this method would be sensitive to a $C P$ violating phase difference of $5^{\circ}$ in the $\phi \pi^{+}$resonance at $90 \%$ confidence level, or a magnitude difference of $11 \%$ in the $\kappa(800) K^{+}$resonance with a significance of $3 \sigma$. However, none of these discrepancies have been found on a data sample of $35 \mathrm{pb}^{-1}$ at $\mathrm{LHCb}$ [29], consisting of approximately 403000 selected candidates.

\subsection{Search for $C P$ violation in $D \rightarrow \pi^{-} \pi^{+} \pi^{+} \pi^{-}$decays}

This $C P$ violation search is a model independent search, similar to the previously described 3-body analysis. The Cabibbo favored $D \rightarrow K \pi \pi \pi$ mode, with a branching fraction about 10 times larger, is used as control channel to study detector and production asymmetries.

The asymmetry significance, obtained from expression (5.12), is evaluated in different bins across the phase space. An adaptive binning technique is used such that all bins contain at least 100 entries. For different number of bins, p-values are computed using

$$
\chi^{2}=\sum_{k}\left(S_{C P}^{k}\right)^{2}
$$

To minimize detector and production asymmetries, equal amounts of LHCb magnet up and down data are used. Also, fiducial cuts are applied to areas with large asymmetry, and the $D^{0}$ meson sample is reweighted to force the same amount of $D^{0}$ and $\bar{D}^{0}$ in each bin of $\eta$ and $p_{t}$.

Rectangular cuts are applied to reconstruct candidates, and a neural network is used to select signal candidates. The same neural network is also used to select candidates in the control channel.

The sensitivity to $C P$ violating effects is obtained from a toy study with events generated from a FOCUS model described in [30], by forcing a relative phase or magnitude difference between the $D^{0}$ and the $\bar{D}^{0}$. This study shows that the method would be sensitive to a $C P$ violating phase difference of $10^{\circ}$ in the $\rho \rho$ resonance and a magnitude difference of $10 \%$ in the $\kappa(800) K^{+}$resonance. However, no $C P$ violation has been observed at $\mathrm{LHCb}$, with a data sample of 180000 $D \rightarrow \pi^{-} \pi^{+} \pi^{+} \pi^{-}$reconstructed candidates. 


\section{Conclusion}

This document has presented searches for $y_{C P}$ and $A_{\Gamma}$ in two-body charm decays, and $C P$ violation searches in $D^{0} \rightarrow h^{+} h^{-}, D^{+} \rightarrow K^{-} K^{+} \pi^{+}$and $D^{0} \rightarrow \pi^{-} \pi^{+} \pi^{+} \pi^{-}$decays. In particular, the latter is the first model-independent search for local $C P$ violation in a charm four-body decay. Sensitivity to charm $C P$ violation and charm mixing in $\mathrm{LHCb}$ has become very promising.

\section{References}

[1] A. Falk et al., Phys. Rev. D 69, 114021 (2004).

[2] E. Golowich et al., Phys. Rev. D 76, 095009 (2007).

[3] Y. Grossman et al., Phys. Rev. D 75, 036008 (2007).

[4] B. Aubert et al., BaBar collaboration, Phys. Rev. Lett. 98, 211802 (2007).

[5] L. M. Zhang et al., Belle collaboration, Phys. Rev. Lett. 96, 151801 (2006).

[6] T. Aaltonen et al., CDF collaboration, Phys. Rev. Lett. 100, 121802 (2008).

[7] B. Aubert et al., BaBar collaboration, Phys. Rev. D 78, 011105(R) (2008).

[8] M. Starič et al., Belle collaboration, Phys. Rev. Lett. 98, 211803 (2007).

[9] B. Aubert et al., BaBar collaboration, Phys. Rev. D 80, 071103 (2009).

[10] B. Aubert et al., BaBar collaboration, Phys. Rev. Lett. 103, 211801 (2009).

[11] B. Aubert et al., BaBar collaboration, Phys. Rev. D 76, 014018 (2007).

[12] U. Bitenc et al., Belle collaboration, Phys. Rev. D 72, 071101(R) (2005).

[13] U. Bitenc et al., Belle collaboration, Phys. Rev. D 77, 112003 (2008).

[14] D. M. Asner et al., CLEO collaboration, Phys. Rev. D 72, 012001 (2005).

[15] L. M. Zhang et al., Belle collaboration, Phys. Rev. Lett. 99, 131803 (2007).

[16] B. Aubert et al., BaBar collaboration, Phys. Rev. Lett. 100, 061803 (2008).

[17] M. Starič et al., Belle collaboration, Phys. Lett. B 670, 190-195 (2008).

[18] B. Aubert et al., BaBar collaboration, Phys. Rev. D 78, 051102 (2008).

[19] K. Arinstein et al., Belle collaboration, Phys. Lett. B 662, 102-110 (2008).

[20] X. C. Tian et al., Belle collaboration, Phys. Rev. Lett. 95, 231801 (2005).

[21] R. Bailey et al. Z. Phys. C 28, 357 (1985).

[22] V. Gligorov, CERN-THESIS-2008-044

[23] R. Aaij et al., LHCb collaboration, JHEP 1204, 129 (2012).

[24] T. Aaltonen et al., CDF collaboration, arXiv:1111.5023 [hep-ex]

[25] I.I. Bigi and A. Paul and S. Recksiegel, JHEP 1106, 089 (2011).

[26] R. Aaij et al., LHCb collaboration, Phys. Rev. Lett. 108, 111602 (2012).

[27] A. Di Canto, La Thuile 2012.

[28] CDF Note 10784.

[29] R. Aaij et al., LHCb collaboration, Phys. Rev. D 84, 112008 (2011).

[30] J. Link et al., Focus collaboration, Phys. Rev. D 75, 052003 (2007). 